\title{
LA REALIMENTACIÓN Y SU INCIDENCIA EN LA SATISFACCIÓN DE LOS ESTUDIANTES EN CAMPUS VIRTUAL
}

\author{
María del Rocío Pabón Jaramillo ${ }^{4}$ y \\ Rubén Darío Múnera Tangarife ${ }^{5}$
}

\begin{abstract}
RESUMEN
El objetivo principal de la presente investigación es identificar y cuantificar las variables que tienen mayor incidencia en los procesos de realimentación y acompañamiento con los estudiantes de la UNAD y proponer la elaboración de planes de mejoramiento que permitan cualificar el desempeño de los tutores y estudiantes en los cursos en campus. Para ello se trabajó sobre dos variables fundamentales; la primera, el nivel de satisfacción de los estudiantes frente al proceso de realimentación que reciben por parte de sus tutores y segunda, la descripción de las características que presentaban dichas realimentaciones. El proyecto se llevó a cabo con una población de 247 estudiantes del CEAD Acacías, que tenían matriculado el curso de Proyecto Pedagógico Unadista en campus, correspondiente al II semestre de 2009, de los cuales se aplicó encuesta al 25.5\% (66 estudiantes).

Para adelantar el anterior proceso se elaboró un cuestionario que dio cuenta del nivel de satisfacción de los estudiantes frente a los procesos de realimentación y a las características de la misma. Los resultados del estudio indican que entre el $60 \%$ y el $70 \%$ de la muestra está satisfecha con dichos procesos pero se deben mejorar los canales de comunicación. Dentro de las características de la realimentación, se hace especial énfasis en ofrecer el valor agregado que potencie la dimensión humana, que requiere fortalecerse a través de la red para hacer más cálida la comunicación y la interacción.
\end{abstract}

Palabras clave: realimentación, satisfacción estudiantil, campus virtual.

\begin{abstract}
This research's main objective is to identify and quantify the variables that have greater impact on processes of feedback and accompanying from students, and to propose the production of improvement plans that allow qualifying the performance of the tutors and students in the courses in campus. For this, it was worked on two fundamental variables: first, the level of students satisfaction regarding the process of receiving feedback from their tutors and second the description of the features that had the feedback offered by tutors to students. The project was carried out with students of Acacias CEAD of the Academic Pedagogic Project Course,

4 Escuela Ciencias de la Educación, CEAD Acacías.

5 Ingeniero Químico CEAD Palmira. Grupo de investigación: Kuwei y Tecnogenesys. Código del grupo en Colciencias. COL0057248. Línea de investigación: Ambientes de Aprendizaje Online, Red de Investigación: Consejería Académica; Inteligencia colectiva y cibersociedad. Nota: Trabajo de investigación en la Maestría Higher Educatión - UNAD Florida.
\end{abstract}


corresponding to the second semester of 2009 , to 247 students, of which only answered the survey $25.5 \%$ (66 students).

To advance in the previous process, it was developed a questionnaire that showed the level of student satisfaction versus feedback processes, and also were reviewed various feedbacks that showed characteristics of them. The results indicate that between $60 \%$ and $70 \%$ of the sample is pleased with the feedback processes, but should improve channels of communication. Inside the characteristics of the feedback, special emphasis is done in offering the added value that promotes the human dimension, which it needs to fortify across the network to make the communication and the interaction better.

Key words: feedback, student satisfaction, virtual campus.

\section{INTRODUCCIÓN}

Cuando nos encontramos en un ámbito como el educativo, donde los usuarios se vinculan a nuestra institución con el interés de recibir el mejor servicio y los mejores beneficios, hay una invitación implícita a revisar los procesos realizados por cada uno de nosotros, para dar respuesta acorde con las necesidades de los estudiantes y de la sociedad actual y futura.

El aprendizaje en ambientes virtuales está determinado por diversas variables; entre ellas, y de las más importantes, es la comunicación que se establece entre tutor- estudiante y viceversa. La comunicación se utiliza especialmente en los procesos de realimentación, la cual puede ser valorada positiva o negativamente por el usuario. Es por ello que este trabajo se ha orientado a revisar estos aspectos en la comunicación escrita, la cual es una herramienta que permite estimular al estudiante en los procesos de aprendizaje en línea.

La cantidad de estudiantes matriculados en cursos virtuales cada día es mayor (UNAD crece en número de estudiantes inscritos, 2009), lo que ocasiona demoras en la asignación de tutores nuevos para guiarlos en su proceso de aprendizaje, en la conformación de grupos de trabajo, y en la ubicación en los enlaces para que puedan responder a través de los foros. Lo anterior genera una situación que conlleva a demoras para contestar los mensajes de los estudiantes y atender sus requerimientos. También es conocido por los tutores que muchos estudiantes no terminan el curso y lo abandonan poco después de haber comenzado, ¿por qué?, por las razones señaladas antes, sin embargo, el interés es centrarnos en los procesos de realimentación e investigar qué está pasando al respecto.

De igual manera, aunque existe una rúbrica de evaluación, es importante determinar cómo se están dando los procesos de realimentación y el nivel de satisfacción de los estudiantes frente a los mismos ya que dentro de las quejas que se suelen formular, se hace referencia a demoras en la realimentación y, en general, a diversas de sus peticiones, de ahí que sea necesario indagar sobre su sentir frente a estos procesos. 
La retroalimentación es un proceso importante que se vivencia al interior de las instituciones educativas, está integrado a los procesos que tienen que ver con la valoración del trabajo del otro y con el alcance de sus competencias. Por tanto, es importante recordar que el objetivo de la retroalimentación debe ser promover la mejora permanente y el deseo de superación, por lo tanto, nos ubicamos ante un gran desafío: incidir en el aprendizaje.

La realimentación es un proceso importante que se vivencia al interior de las instituciones educativas y está integrado a los procesos que tienen que ver con la valoración del trabajo del otro y con el alcance de sus competencias. Por lo tanto, es importante recordar que su objetivo debe ser promover la mejora permanente y el deseo de superación. De esta manera, nos ubicamos ante un gran desafío: incidir en el aprendizaje.

La realimentación forma parte del proceso que permite identificar errores, corregir aspectos y orientar adecuadamente frente a situaciones específicas. Requiere, por parte de quien la brinda, conocimientos y habilidades que posibiliten una mejor entrega de la información y así generen una percepción positiva de la misma por parte de quien la recibe.

Jané (2005) indica que desde los años sesenta los paradigmas sobre evaluación han ido cambiando. Considera numerosos los autores que sostienen que la manera como los estudiantes son evaluados tiene una directa influencia en la calidad de su aprendizaje, por eso se plantean procedimientos menos jerárquicos, que replantean las relaciones de poder.

Ramírez (2007) adelantó un estudio sobre retroalimentación en ambientes virtuales en el Tecnológico de Monterrey. Este estudio estuvo dirigido al impacto de la retroalimentación en los cursos virtuales, como parte esencial entre la comunicación profesor-alumnos. La investigación se aplicó en cursos en línea y los participantes fueron maestros y alumnos y se indagó sobre las diversas sesiones de comunicación entre estos. Los hallazgos más relevantes fueron: la retroalimentación de tareas, actividades y foros de discusión, proporcionan no sólo aspectos cognitivos o revisión de contenidos, para las mejoras en el desarrollo académico, sino que tiene un impacto a nivel psicológico, actitudinal que favorece la motivación, interés y atención por parte del alumno, visualizando la aplicación de dichos contenidos en su vida profesional.

El impacto que genera la retroalimentación, la actitud y dirección del mensaje que propicia el docente a través de las Nuevas Tecnologías de la Información y la Comunicación (NTICS), contribuyen a las expectativas del alumno frente al curso, para lograr un aprendizaje significativo. Los resultados de la investigación estuvieron dirigidos a elaborar principios básicos para realizar una retroalimentación exitosa: empatía, motivación, asertividad y contenido, y la finalidad de la retroalimentación, en el sentido de guiar, sugerir ideas y planes y evitar mensajes imperativos.

Flores (2006) presenta el resultado de un proyecto de investigación realizado entre 1999 y 2002 que buscaba revisar si existían diferencias entre un profesor virtual y uno tradicional. El trabajo de Flores es interesante ya que aporta dos hallazgos relevantes: el tipo de trabajo que realiza un profesor "virtual" es muy diferente y no una simple extensión del "real", asimismo, es un trabajo que los maestros "reales" están mal equipados para realizar. La mayor parte del tiempo el docente 
"virtual" se dedica a dos actividades que se identifican como "redacción epistolar" y "enseñanza holográfica", la primera hace referencia a la forma como el tutor se relaciona con el estudiante y la segunda, a que la enseñanza deja de ser una tarea individual y pasa a ser colectiva, en el sentido que son diversas personas las que interactúan con los estudiantes.

Reconocer que ser un profesor virtual implica realizar un trabajo radicalmente distinto que supone aprender a comunicarse con alumnos individualmente y por escrito, y trabajar con otros especialistas de manera diferente, sería un primer paso indispensable para participar en ese proceso.

Chaux (2008) especifica: "Algunos profesores de colegio y de universidad justifican su fuerte y muchas veces maltratante retroalimentación con el argumento de que no se puede ser suave con los estudiantes porque, de otra forma, no aprenderían. Detrás de esta creencia hay la falsa visión de que hay sólo dos alternativas: ser suave y permisivo con los estudiantes, o ser fuerte y duro. En cambio, es perfectamente posible ser exigente y cuidadoso al mismo tiempo. Son dos dimensiones distintas. De hecho, lo más efectivo en términos de aprendizaje es combinar las dos". De igual manera sostiene: "La retroalimentación que podemos dar como profesores universitarios o de colegio en las evaluaciones a nuestros estudiantes tiene un impacto mucho mayor del que usualmente somos conscientes. Y aquí es claro que la forma es tan importante como el contenido. El mismo contenido puede generar motivación o desmotivación dependiendo de cómo lo digamos".

Los aspectos referidos permiten tener claridad en que los procesos de retroalimentación son fundamentales en las actividades en línea y que ello implica revisar la manera en que se está haciendo este porceso al interior de la UNAD. Como lo indica Mella, Gutiérrez y Maurize (citado por Trisca, 2009), es fundamental para mantener un grado de interés y motivación en los alumnos, estar atentos a los diferentes estilos de comunicación. De igual manera, Meza (2004) indica que una de las funciones del tutor es la de mantener la motivación de los estudiantes.

Teniendo comoreferentes los aspectos anteriores, se formularon dos preguntas de investigación: ¿De qué manera la realimentación que ofrecen los tutores en campus virtual, incide sobre el nivel de satisfacción de los estudiantes del curso Proyecto Pedagógico Unadista del CEAD Acacías?, ¿Cuáles son las características que presentan las realimentaciones ofrecidas por los tutores en el curso de Proyecto Pedagógico Unadista de los estudiantes del CEAD Acacías?

Para Nadeau G. (1994) una Institución de Educación Superior de calidad es aquella que tiene un sistema de evaluación y realimentación de información sobre la formación del estudiante. Ello permite practicar los ajustes apropiados en los programas o las políticas cuando se plantea la necesidad de cambio o de mejoramiento.

Desde esta óptica, es necesario conceptualizar que el proceso de realimentación está vinculado a los procesos de mejoramiento de la calidad. Nunca se sabe cómo se están adelantando los procesos, ni qué tanto son aceptados por los usuarios de los mismos, hasta que ellos indiquen; 
por tanto, revisar estas prácticas pedagógicas, objeto de este trabajo, resulta ser un ejercicio vital para las transformaciones que continuamente deben darse.

Algunos autores manifiestan que la retroalimentación, debe iniciar siempre por resaltar los aspectos positivos y presentar lo negativo como algo que se puede mejorar; en lo posible identificar a la persona por su nombre, especialmente en los ambientes virtuales y referirse siempre al producto, puesto que la persona tiene las potencialidades y la capacidad para hacer bien las cosas.

Si como dice Sarmiento (2008) "la evaluación es un proceso permanente y continuo de indagación y valoración de una propuesta educativa. Su finalidad es producir información que alimente la toma de decisiones oportunas y pertinentes que garanticen la eficiencia, la eficacia y la calidad de procesos, resultados e impactos en el mejoramiento social. No es control ni fiscalización, es un proceso que permite a los actores involucrados aprender y adquirir experiencia de lo planificado y ejecutado para optimizar los resultados de su gestión”.

Ésta entonces debe ser presentada positivamente, reconociendo las posibilidades de mejora que tienen los participantes, además, invitarlos a desarrollar su potencial y habilidades. Siendo así, en esta búsqueda de la mejora, la retroalimentación es un enlace importante para potenciar, favorecer y concientizar sobre los procesos y aprendizajes, es un vínculo que estrecha lazos y posibilita desarrollos.

\section{OBJETIVO GENERAL}

Identificar y cuantificar las variables que tienen mayor incidencia en los procesos de realimentación y de acompañamiento de los estudiantes para proponer la elaboración de planes de mejoramiento que permitan cualificar el desempeño de tutores y estudiantes en el curso de Proyecto Pedagógico Unadista del CEAD Acacías y de los cursos virtuales que ofrece la UNAD.

\section{OBJETIVOS ESPECÍFICOS}

Identificar los aspectos que los estudiantes consideran como de mayor importancia dentro del proceso de realimentación.

Cuantificar la satisfacción de los estudiantes frente a los procesos de realimentación.

Determinar las características principales de la realimentación que se da a los estudiantes del Cead Acacías que estudian el curso de Proyecto Pedagógico Unadista.

Encontrar algunos aspectos que puedan ser ajustados para que la realimentación se constituya en un elemento de estímulo y motivación para el estudiante dentro de su proceso de aprendizaje. 


\section{METODOLOGÍA}

El trabajo de investigación realizado fue de tipo descriptivo. Se aplicó un instrumento que dio cuenta del grado de satisfacción de los estudiantes del curso de Proyecto Pedagógico Unadista, frente a los procesos de realimentación ofrecido por los tutores en campus y se revisaron diversas realimentaciones, estableciendo las características que reunían éstas.

Las variables que se midieron en el estudio fueron:

- El nivel de satisfacción de los estudiantes frente al proceso de realimentación que reciben por parte de sus tutores.

- Las características que presentan las realimentaciones ofrecidas por los tutores a sus estudiantes.

Participantes: como ya se mencionó, la población correspondió a 247 estudiantes que tenían matriculado el curso de Proyecto Pedagógico Unadista en campus, pertenecientes al CEAD de Acacías, de estos 66 respondieron la encuesta, que representa el 25.5\%.

Instrumentos: los principales instrumentos utilizados fueron la encuesta para medir el nivel de satisfacción estudiantil y la revisión de las realimentaciones frente a las cuales se establecieron 10 categorías.

Procedimiento:

- Se elaboró un cuestionario con preguntas cerradas que permitió cuantificar el nivel de satisfacción de los estudiantes frente a los procesos de realimentación.

- Se revisaron diversas realimentaciones ofrecidas por los tutores para determinar las características, establecer las categorías encontradas y realizar el análisis respectivo. Para ello se revisaron las realimentaciones ofrecida por 30 tutores en los foros de reconocimiento y foro de trabajo colaborativo 1.

- Se tabuló la información y se elaboraron gráficos que ilustran el comportamiento de variable.

- Se elaboraron conclusiones y se recomendaron futuros trabajos.

Análisis estadístico: se utilizó Excel como software para el manejo de los datos y se elaboraron gráficos para ilustrar el comportamiento de la primera variable estudiada. Frente a la segunda variable, se establecieron las categorías y se procedió a describir lo encontrado.

\section{RESULTADOS}

En cuanto a la variable sobre el nivel de satisfacción, a continuación se ubican los resultados organizados en dos grupos. Un primer grupo de preguntas relacionadas con la satisfacción del proceso de realimentación y un segundo grupo de preguntas relacionadas con las características de la realimentación y la satisfacción frente a estas. 
Tabla 1. Preguntas realizadas que se relacionan con la satisfacción de la realimentación

\begin{tabular}{|l|c|c|c|c|c|}
\hline Preguntas realizadas que se relacionan con la satisfacción de la realimentación & \multicolumn{4}{|c|}{ Opción de respuesta, $\%$} \\
\hline $\mathbf{1}$ = nunca; $\mathbf{2}=$ casi nunca; $\mathbf{3}=$ algunas veces; $\mathbf{4}$ = casi siempre; $\mathbf{5}$ siempre & $\mathbf{1}$ & $\mathbf{2}$ & $\mathbf{3}$ & $\mathbf{4}$ & $\mathbf{5}$ \\
\hline Me siento satisfecho con el proceso de realimentación ofrecido: & $6 \%$ & $8 \%$ & $21 \%$ & $27 \%$ & $38 \%$ \\
\hline La realimentación que obtengo me motiva a continuar en el proceso: & $5 \%$ & $8 \%$ & $17 \%$ & $27 \%$ & $43 \%$ \\
\hline Generalmente estoy de acuerdo con la realimentación que me entregan: & $5 \%$ & $2 \%$ & $32 \%$ & $24 \%$ & $38 \%$ \\
\hline Considero que la realimentación es significativa en mi aprendizaje: & $2 \%$ & $2 \%$ & $14 \%$ & $17 \%$ & $65 \%$ \\
\hline Me siento desmotivado con la realimentación que recibo del tutor: & $32 \%$ & $16 \%$ & $29 \%$ & $16 \%$ & $8 \%$ \\
\hline Me he sentido acompañado por el tutor dentro de esta dinámica de trabajo: & $5 \%$ & $16 \%$ & $18 \%$ & $29 \%$ & $32 \%$ \\
\hline La forma de comunicación del tutor debe mejorar: & $5 \%$ & $14 \%$ & $32 \%$ & $22 \%$ & $27 \%$ \\
\hline
\end{tabular}

Tabla 2. Preguntas realizadas que se relacionan con las características de la realimentación

\begin{tabular}{|l|c|c|c|c|c|}
\hline \multicolumn{1}{|c|}{$\begin{array}{c}\text { Preguntas realizadas que se relacionan con las características de la } \\
\text { realimentación }\end{array}$} & \multicolumn{3}{|c|}{ Opción de respuesta, \% } \\
\hline $\mathbf{1}$ = nunca; $\mathbf{2}=$ casi nunca; 3 = algunas veces; $\mathbf{4}$ = casi siempre; 5 siempre & $\mathbf{1}$ & $\mathbf{2}$ & $\mathbf{3}$ & $\mathbf{4}$ & $\mathbf{5}$ \\
\hline Recibo realimentación oportuna por parte de mi tutor: & $0 \%$ & $12 \%$ & $23 \%$ & $23 \%$ & $42 \%$ \\
\hline La realimentación contempla aspectos numéricos y también descriptivos: & $6 \%$ & $5 \%$ & $30 \%$ & $29 \%$ & $30 \%$ \\
\hline La realimentación resalta mis fallas: & $6 \%$ & $5 \%$ & $30 \%$ & $38 \%$ & $21 \%$ \\
\hline La realimentación reconoce mis aciertos y me plantea qué debo mejorar: & $5 \%$ & $3 \%$ & $17 \%$ & $38 \%$ & $37 \%$ \\
\hline En la realimentación el tutor tiene en cuenta la rúbrica evaluativa: & $6 \%$ & $5 \%$ & $13 \%$ & $32 \%$ & $44 \%$ \\
\hline La realimentación está centrada en el trabajo presentado: & $5 \%$ & $3 \%$ & $13 \%$ & $35 \%$ & $44 \%$ \\
\hline El tutor está dedicando buen tiempo a ofrecer la realimentación: & $5 \%$ & $10 \%$ & $30 \%$ & $23 \%$ & $33 \%$ \\
\hline La forma de comunicación del tutor es agradable y cálida: & $3 \%$ & $5 \%$ & $22 \%$ & $27 \%$ & $43 \%$ \\
\hline La realimentación propicia un espacio de crecimiento para mi: & $2 \%$ & $2 \%$ & $17 \%$ & $33 \%$ & $46 \%$ \\
\hline
\end{tabular}

Frente a la variable relacionada con las características de la realimentación, se presenta a continuación un cuadro resumen de las realimentaciones revisadas.

Tabla 3. Número de foros y mensajes revisados

\begin{tabular}{|c|c|c|c|c|c|c|c|}
\hline \multicolumn{2}{|c|}{ Número de estudiantes } & \multicolumn{2}{|c|}{ Número de realimentaciones } & \multicolumn{2}{c|}{ Número de tutores } & \multicolumn{2}{c|}{$\begin{array}{c}\text { Intercambio de mensajería } \\
\text { tutor-estudiante }\end{array}$} \\
\hline Hombre & Mujer & $\begin{array}{c}\text { Foro de } \\
\text { reconocimiento }\end{array}$ & $\begin{array}{c}\text { Foro de trabajo } \\
\text { colaborativo uno }\end{array}$ & Hombre & Mujer & No & $\mathrm{Si}$ \\
\hline 14 & 16 & 30 & 30 & 10 & 20 & 11 & 19 \\
\hline
\end{tabular}

De acuerdo con las realimentaciones revisadas se establecieron 10 categorías las cuales se presentan a continuación. 
Tabla 4. Categorización de la realimentación

\begin{tabular}{|c|c|c|}
\hline $\begin{array}{l}\text { Identificación de } \\
\text { la categoría }\end{array}$ & Categorización de la realimentación & Descripción \\
\hline 1 & Usa la rúbrica de evaluación & $\begin{array}{l}\text { Toma en cuenta los datos suministrados en la rúbrica } \\
\text { de forma clara. }\end{array}$ \\
\hline 2 & Reconoce los avances & $\begin{array}{l}\text { Resalta los aspectos positivos del trabajo elaborado, } \\
\text { reconociendo los avances. }\end{array}$ \\
\hline 3 & $\begin{array}{l}\text { Expone lo negativo como susceptible } \\
\text { de mejora }\end{array}$ & $\begin{array}{l}\text { Indica los aspectos en los cuales se falló, manifestando } \\
\text { la posibilidad de mejora frente a los procesos. }\end{array}$ \\
\hline 4 & Motiva al estudiante & $\begin{array}{l}\text { Estimula a continuar en el proceso, reconoce que los } \\
\text { ejercicios son parte de la mejora. Impulsa a seguir } \\
\text { trabajando y construyendo. }\end{array}$ \\
\hline 5 & Muestra empatía & $\begin{array}{l}\text { Inicia con un saludo. Identifica por el nombre haciendo } \\
\text { el proceso más personal, felicita. }\end{array}$ \\
\hline 6 & Orienta & $\begin{array}{l}\text { Aclara los aspectos que debe mejorar y lo remite a } \\
\text { consultar temas concretos. }\end{array}$ \\
\hline 7 & Usa mensajes imperativos & $\begin{array}{l}\text { Indica las fallas, remite a revisar el material, sin establecer } \\
\text { empatía, indica que esperaba más y personaliza. }\end{array}$ \\
\hline 8 & Es asertiva & $\begin{array}{l}\text { Da cuenta del contenido del trabajo, especificando temas } \\
\text { concretos o frases utilizadas, las cuales debe revisar. } \\
\text { Específica tema por tema; conoce el trabajo y, por tanto, } \\
\text { se remite al mismo. }\end{array}$ \\
\hline 9 & Reclama por el trabajo en equipo & $\begin{array}{l}\text { Plantea el trabajo en equipo como excusa para bajar } \\
\text { puntos, sin mayor claridad y sin especificar otros } \\
\text { aspectos. }\end{array}$ \\
\hline 10 & Es genérica & $\begin{array}{l}\text { No se especifica nada concreto del trabajo presentado. Se } \\
\text { incluye dentro del grupo denominado genérico, porque } \\
\text { lo que se dice no da cuenta del contenido. Aunque puede } \\
\text { tener elementos motivacionales y de empatía. }\end{array}$ \\
\hline
\end{tabular}

\section{DISCUSIÓN}

Con relación a la satisfacción de los estudiantes frente a la realimentación, se encontró que se sienten satisfechos (65\%), son motivados por la realimentación $(70 \%)$, están de acuerdo con la realimentación $(62 \%)$, la realimentación es significativa en su proceso de aprendizaje (82\%), nunca se han sentido desmotivados por la realimentación (48\%), se han sentido acompañados por su tutor (62\%) y consideran que la forma de comunicación con su tutor debe mejorar (49\%).

De acuerdo con las características de la realimentación y su satisfacción, se encontró que los estudiantes observan que la realimentación es oportuna (65\%), contempla aspectos numéricos y descriptivos (59\%), resalta las fallas de los estudiantes $(59 \%)$, reconoce los aciertos y le plantea que debe mejorar (75\%), el tutor tiene en cuenta la rúbrica de evaluación $(76 \%)$, la realimentación está centrada en el trabajo presentado (79\%), el tutor dedica un buen tiempo para ofrecer la realimentación (56\%) y la forma de comunicación del tutor es agradable y cálida $(70 \%)$. 
En cuanto a las características de la realimentación, basada en la revisión que se hizo de las mismas, es de indicar que se identificaron dos formas como se ofrece por parte de los tutores; una "basada en la rúbrica evaluativa" y, la otra, "basada en aspectos generales" que no abordan los temas contemplados en la rúbrica evaluativa.

Si bien estas dos formas ofrecen elementos motivacionales y de empatía en forma general, es necesario valorar que la realimentación basada en la rúbrica de acuerdo con los criterios planteados en la guía de actividades, es indispensable mantenerla, ya que ofrece criterios claros, que con antelación son conocidos por el estudiante, quien haciendo uso de su proceso de aprendizaje autónomo podrá utilizar para valorar previamente su trabajo.

Revisamos las dos posibilidades que se ofrecen desde la realimentación "basada en la rúbrica"; la primera, enfocada netamente en el cuadro de la rúbrica, lo que lleva a cambio de valores o datos numéricos y la segunda, basada en una descripción que recoge los aspectos de la rúbrica. Es de indicar, que basarse en el formato exclusivamente para cambiar sólo los datos cuantitativos, sin adicionar elementos indispensables que fortalezcan la relación entre tutor-estudiante no le dan el valor agregado que potencia la dimensión humana que requiere fortalecerse a través de la red para hacer más cálida la comunicación y la interacción, fortaleciendo a través de la misma la satisfacción y permanencia de los estudiantes en la universidad. La realimentación basada en la descripción que recoge los aspectos de la rúbrica, tiende a ser más personalizada y a recoger diversos elementos que la hacen más cercana.

La realimentación "basada en los aspectos generales", que corresponde a la otra forma de presentación utilizada por los tutores del curso Proyecto Pedagógico Unadista, si bien cuenta con varios aspectos que la caracterizan como son, por un lado, la motivación, la empatía, y por el otro, estar orientada hacia la dificultad o deficiencia que presenta el trabajo del estudiante; debe de ajustarse en el sentido de incluir los aspectos de la rúbrica y tener en cuenta que en las ocasiones en las que el producto es valorado positivamente, ejemplo, "José, muy buen trabajo", también requiere que se mencionen y especifiquen los aspectos positivos y no dar por hecho que se sobreentiende que todo los puntos fueron alcanzados con el máximo puntaje, ya que el estudiante requiere fortalecer su autoestima, que le reconozcan su trabajo no sólo con una nota cuantitativa sino con una descripción cualitativa que dé cuenta de su proceso y fortalezas detectadas.

Los aspectos revisados y que se muestran fortalecidos en los procesos de realimentación hacen referencia a la motivación y la empatía. Sin embargo, aunque esto se hace evidente, es importante hacer énfasis en la personalización, ya que muy seguramente por la necesidad de abreviar y facilitar el proceso de evaluación, los tutores (consejeros), reemplazan los nombres por formas genéricas como "apreciado estudiante", entre otras.

Llama la atención la respuesta sobre si la realimentación resalta las fallas ya que un $58 \%$ en la encuesta considera que sí y un 30\% lo considera presente algunas veces. Esta situación nos indica que los aspectos encontrados en los tipos de realimentación genérica detectados, 
en donde se omite la rúbrica y se destacan los aspectos en los cuales están las fallas, dejando de lado, los aspectos positivos, muestra que es necesario, así el producto esté óptimo en casi todos los ítems de la rúbrica, nombrarlos y reconocer su buen desarrollo, para evitar el concepto negativo, centrado en las fallas que percibe el estudiante. Además de tener cuidado con estimular constantemente al estudiante, pues no se trata de omitir ni los aciertos, ni los errores, sino reconocerlos y exponerlos de manera que estimulen al estudiante a avanzar en sus procesos.

Otro aspecto a revisar y que no coincide con el análisis de las realimentaciones es la respuesta dada por los estudiantes frente al ítem, "en la retroalimentación el tutor tiene en cuenta la rúbrica", donde el $76 \%$ considera que sí y sólo el porcentaje restante indica lo contrario; es de aclarar que en la revisión de las realimentaciones, se encontraron dos tipos en igual proporción, una centrada en la rúbrica y otra en la generalidad.

Los ítems enfocados en conocer si la realimentación fue centrada en el trabajo, o si el tutor dedicaba buen tiempo a ofrecerla y si el acompañamiento había sido positivo han sido valorados por encima del 50\% y dan cuenta del buen acompañamiento brindado por los tutores en el curso de PAP. Sin embargo, es importante revisar las insatisfacciones, las cuales podemos centrar en las características de la retroalimentación como forma de elevar el grado de satisfacción, ya que posiblemente este descontento esté relacionado con realimentaciones generalizadas, o rígidas centradas en la rúbrica, que no dan mayor posibilidad de generar empatía y motivar al estudiante en su proceso. Por tanto, es bueno considerar el ítem que indaga sobre si la forma de retroalimentación debe mejorar, en el cual los estudiantes en forma abierta están dispuestos al cambio y a la mejora permanente y un $43 \%$ indica la necesidad de cambiar, lo que conlleva a estar permanentemente revisando estos procesos y haciendo las mejoras respectivas.

\section{CONCLUSIONES}

De acuerdo con los resultados obtenidos en la investigación, se puede concluir que el nivel de satisfacción con la realimentación es ampliamente positivo, oscila entre $60 \%$ y $70 \%$. Los mayores porcentajes de las respuestas se encuentran en siempre y casi siempre. Sin embargo, un grupo pequeño entre un $2 \%$ y un $5 \%$ respondió que nunca estaba complacido con alguna de las preguntas relacionadas con la satisfacción en la realimentación. Este porcentaje aunque pequeño resulta preocupante y es necesario que la Universidad identifique claramente lo que puede estar sucediendo con estos estudiantes.

Lo encontrado en este estudio, frente a la revisión de los procesos de realimentación tanto en sus características como en el grado de satisfacción de los estudiantes, es positivo, sin embargo, no hay que perder de vista que este curso es manejado por consejeros quienes en forma general han venido siendo formados para cautivar a los estudiantes, estar atentos a ellos, orientarlos en sus procesos y facilitar su permanencia en la institución.

Se detectaron en forma general dos tipos de realimentación ofrecidos por los tutores; una centrada en la rúbrica de evaluación y la otra, en aspectos generalizados que no abordan la 
rúbricapero que consolidan en su interior aspectos esenciales que estimulan la motivación y la empatía, elementos importantes para los estudiantes.

Si bien los procesos de retroalimentación deben centrarse en la rúbrica evaluativa, ésta debe complementarse con las posibilidades que brinda la comunicación, dirigiendo especialmente cualquier orientación a motivar a los estudiantes, a generar empatía con ellos y a reducir la distancia a través del diálogo fluido y orientador que se puede ofrecer en línea.

Es importante tener en cuenta que ante la presentación de productos por parte de los estudiantes, que cumplen con las diferentes variables expuestas en la rúbrica evaluativa, debe considerarse también la explicación de cada punto, reconociendo la buena elaboración de los ítems; de esta manera, no quedan en la realimentación sólo los aspectos a mejorar, ya que esto, a pesar de que el estudiante tenga una buena nota, da la sensación que el tutor se centra básicamente en ellos.

Es necesario continuar este trabajo de investigación con el siguiente grupo de estudiantes que curse Proyecto Pedagógico Unadista con el fin de hacer otra evaluación similar a la realizada en este informe y evaluar tendencias de las respuestas. Además de participar los resultados de la investigación a los tutores del curso Proyecto Pedagógico Unadista con el fin de que se enteren de los hallazgos encontrados y que se propongan planes de mejoramiento.

De igual manera, es importante formular trabajos de investigación que permitan identificar lo que puede estar sucediendo con los estudiantes que muestran un alto nivel de inconformidad y capacitar al personal académico en general sobre la influencia que tienen los procesos de realimentación en los estudiantes y generar conversatorios o espacios que permitan compartir experiencias, valorar estos procesos y construir formas adecuadas de trabajar y realimentar a los estudiantes, buscando cumplir con el objetivo esencial de la educación, la cual va dirigida a incentivar el aprendizaje, aumentar el interés, reconocer los aciertos y dificultades, y especialmente aportar al desarrollo de cada estudiante.

Finalmente, consideramos necesario evaluar otros cursos que sean dirigidos por tutores y no por consejeros, quienes si bien deben estar capacitados para ofrecer un buen servicio y estimular la permanencia de los estudiantes en su curso y en la institución, posiblemente su realimentación reúna otras características particulares.

\section{REFERENCIAS BIBLIOGRÁFICAS}

CHAUX, E. (2008), Retroalimentar y Crecer. Periódico Altablero, el periódico de un país que educa y que se educa. Núm. 44. Recuperado el 4 de octubre 4 de 2009. http://www. mineducacion.gov.co/1621/article-162349.html.

FLORES,E.(2006), Encontrandoal ProfesorVirtual. Resultado de un Proyecto de Investigación Acción. Revista Mexicana de investigación educativa. Pág. 91-128. Recuperado el 4 de octubre 4 de 2009. http://redalyc.uaemex.mx/redalyc/pdf/140/14002806.pdf. 
MARC, J. (2005), Evaluación del Aprendizaje: ¿Problema o Herramienta? En publicación: Revista de Estudios Sociales, No. 20. CESO, Centro de Estudios Socioculturales e Internacionales, Facultad de Ciencias Sociales, Universidad de Los Andes, Bogotá: Colombia. Recuperado el 4 de octubre 4 de 2009. http://redalyc.uaemex.mx/src/inicio/ ArtPdfRed.jsp?iCve=81502007\&iCveNum $=8140$.

RAMÍREZ, S. (2007), Realimentación en Ambientes Virtuales. Escuela de Graduados en Educación del Tecnológico de Monterrey. Recuperado el 4 de octubre 4 de 2009. http://rediien.comitenorte.org. $\mathrm{mx} /$ index.php?option $=$ com_docman\&task $=$ doc download\&gid=23\&Itemid=24.

SALINAS, M.L. El Sentido de la Evaluación en las Facultades de Educación. Decana Facultad de Educación. Universidad de Antioquia. Ascofade. Recuperado el 4 de octubre 4 de 2009. http://ascofade.com.co/?q=node/1275.

SARMIENTO, V.M. (2008), Una Mirada desde la Educación Superior. Periódico Altablero, el periódico de un país que educa y que se educa. Núm. 44. Recuperado el 4 de octubre 4 de 2009. http://www.mineducacion.gov.co/1621/article-162368.html.

TRISCA, J.O. La Motivación en los Cursos en Línea. Obtenido octubre 4, 2009. http://trisca. co.cc/textos/motivation.pdf.

(2008). Evaluación para los Aprendizajes. Periódico Altablero, el periódico de un país que educa y que se educa. Núm. 44. Recuperado el 4 de octubre 4 de 2009. http://www. mineducacion.gov.co/1621/article-162342.html.

CIRCULAR NO.0 010. Alertas Tempranas para Cursos Virtuales. Consejería Virtual, (2009). Documento interno, Universidad Nacional Abierta y a Distancia.

COLOMBIA TIENE SU MEGA UNIVERSIDAD. (2009), Periódico El Colombiano. Recuperado el 9 de octubre de 2009. http://www.mineducacion.gov.co/observatorio/1722/ article-197423.html.

UNAD CRECE EN NÚMERO DE ESTUDIANTES INSCRITOS. Recuperado el 9 de octubre de 2009. http://www.alcaldianeiva.gov.co/portalcms/index.php?option=com_c ontent\&view $=$ article $\& i d=1071 \% 3$ Aunad-crece-en-numero-de-universitariosinscritos \&catid $=21 \% 3$ Aen-otras-palabras \&Itemid $=71$. 